\title{
Effect of Edible Coating and Packaging on Physiological and Sensory Attributes of Litchi (Litchi chinensis Sonn.) Fruits
}

\author{
Shubham, N. K. Mishra, Ratna Rai, Ankit Dongariyal, \\ Ravi Kumar* and Tribhuwan Pratap
}

Department of Horticulture, College of Agriculture, Govind Ballabh Pant University of Agriculture and Technology, Pantnagar, U. S. Nagar, Uttarakhand, India

*Corresponding author

\section{A B S T R A C T}

\begin{tabular}{l} 
K e y w or d s \\
$\begin{array}{l}\text { Edible Coating and } \\
\text { Packaging, Litchi, } \\
\text { Litchi chinensis }\end{array}$ \\
\hline Article Info \\
\hline $\begin{array}{l}\text { Accepted: } \\
\text { 07 October } 2020 \\
\text { Available Online: } \\
10 \text { November } 2020\end{array}$ \\
\hline
\end{tabular}

\section{Introduction}

Litchi (Litchi chinensis Sonn.) is an important sub-tropical fruit with high commercial value in the local as well as international market. However, once detached from the tree, the fruit loses its qualities, including sweet and juicy flesh and attractive bright red pericarp colour, within a couple of days under ambient storage temperatures. The short storage life of litchi limits the marketing of the litchi fruit and has become one of the major constraints in litchi industry, where a huge production happens in a short season from mid-May to early July (Huang, 2002). Generally, rapid cooling after harvest and storage at low temperature are one of the most prevalent methods for maintaining the appearance and quality of litchi fruit. It means that the ambient temperature is one of the most important factors affecting the litchi fruit commercial value (Lin et al., 2011). Harvested litchi fruits are a living organism which breaths continuously during storage process. The respiration produces the bio-heat and causes the rise of temperature of fruit group that accelerates water loss and browning (Baldwin et al., 1995). Postharvest browning of litchi fruit was generally thought to be a rapid degradation of anthocyanins 
caused by polyphenol oxidase, producing brown by-products (Jiang, 2000). Postharvest treatments, including Sulphur fumigation and acid dip can effectively inhibited polyphenol oxidase activity and thus delay loss of red pericarp colour of litchi fruit (Zauberman et al., 1991). Therefore, there is a need for alternative novel practices to control respiration rate of the produce which automatically reduces the bio-heat and the resulting fruit temperature change during handling, distribution and retail sale without toxic effects in harvested litchi fruits (Jiang et al., 2003). Application of edible coatings is promising to improve the quality and extend shelf life of produce. There are several advantages associated with edible coatings like, they may be eaten by the consumers along with food, and their use could reduce the waste and solve the solid disposal problem. Besides, they also enhance the organoleptic, mechanical or nutritional properties of fruit, and they can reduce the cost by utilizing byproducts. Attempts to reduce crop losses and maintaining the quality of fresh fruit over a long period of time are a priority for all the producers of horticultural crops.Keeping in views the importance and perish ability nature of litchi fruits, an attempt has been made in the present study to evaluate the effect of packing and edible coating on shelf life and physical characteristics of litchi fruits by applying Xanthan gum, guar gum and methylcellulose as coating materials.

\section{Materials and Methods}

The present experiment was carried out in the Post-graduate Laboratory, Department of Horticulture, GBPU\&T Pantnagar, U.S. Nagar, Uttarakhand. The fruits were collected from HRC, Patharchatta located at the NorthWest Plains of Tarai region of Uttarakhand at an altitude of 243.83 meters above the mean sea level. There was a total of 17 treatments combination i.e. without coating + without packaging material $\left(\mathrm{T}_{0}\right)$; Without coating + Perforated brown paper bag $\left(\mathrm{T}_{1}\right)$; Guar Gum $0.5 \%\left(\mathrm{~T}_{2}\right)$; Guar Gum 1.0\% $\left(\mathrm{T}_{3}\right)$; Guar Gum $1.5 \%\left(\mathrm{~T}_{4}\right)$; Guar Gum 2.0\% ( $\left.\mathrm{T}_{5}\right)$; Xanthan Gum $0.5 \%\left(\mathrm{~T}_{6}\right)$; Xanthan Gum $1.0 \%\left(\mathrm{~T}_{7}\right)$; Xanthan Gum 1.5\% ( $\left.\mathrm{T}_{8}\right)$; Xanthan Gum 2.0\% ( $\mathrm{T}_{9}$ ); Methyl Cellulose (low viscosity) $0.5 \%$ $\left(\mathrm{T}_{10}\right)$; Methyl Cellulose (low viscosity) $1.0 \%$ $\left(\mathrm{T}_{11}\right)$; Methyl Cellulose (low viscosity) $1.5 \%$ $\left(\mathrm{T}_{12}\right)$; Methyl Cellulose (low viscosity) $2.0 \%$ $\left(\mathrm{T}_{13}\right)$; Methyl Cellulose (high viscosity) $0.5 \%$ $\left(\mathrm{T}_{14}\right)$; Methyl Cellulose (high viscosity) $1.0 \%$ $\left(\mathrm{T}_{15}\right.$ ); Methyl Cellulose (high viscosity) $1.5 \%$ $\left(\mathrm{T}_{16}\right)$ and Methyl Cellulose (high viscosity) $2 \%\left(\mathrm{~T}_{17}\right)$ with 3 replication. Xanthan and guar gum solutions were prepared as per method given by Ruelas-Chacón et al., 2017. The methyl cellulose solution (both low viscosity and high viscosity) was prepared by solubilizing the methyl cellulose powder in a mixture of water and ethyl alcohol (2:1) at 75 ${ }^{\circ} \mathrm{C}$ in a high-speed mixer (900 rpm) for 15 minutes.

In all the treatments, glycerol (1\%) was added as plasticizer and ascorbic acid (1\%) as an antioxidant. After uniform application of all the coating materials, fruits were placed in the perforated brown paper bags. There after packaged fruits were stored at low temperature $\left(4^{\circ} \mathrm{C} \pm 1^{\circ} \mathrm{C}\right)$ and $85-90 \%$ related humidity in a refrigerator for further studies. Observations were recorded at two days interval $\left(0,2,4,6,8,10\right.$ and $12^{\text {th }}$ day). Fruit length and breadth was measured with the help of digital Vernier calipers. The water displacement method was used to measured volume of the litchi fruits. On initial day fruits were weighed on an electronic weighing balance and were reweighed at 2 days interval. The weight loss was determined and expressed as percent loss from initial weight. Organoleptic evaluation was carried out on the basis of fruit appearance (colour), taste and flavor. It was assessed on the basis of scoring, as per the hedonic scale ranging from 
9 to 1 , where 9 being the most favorable one noted as "like extremely" and 1 being the least acceptable determined as "dislike extremely". The data was analyzed on two factorial completely randomized design (Factorial C.R.D.) as described by Snedecor and Cochran (1987).

\section{Results and Discussion}

\section{Physiological loss in weight (\%)}

In the present experiment, it was observed that packaging and edible coatings significantly affected the physiological loss in weight with the advancement of the storage duration (Table 1). The maximum loss in the physiological weight $(7.17 \%)$ was recorded in $\mathrm{T}_{0}$ i.e. fruits without coating and packaging, followed by $\mathrm{T}_{1}$ i.e. fruits without coating and packed in perforated brown paper bag $(7.08 \%)$; whereas, the minimum physiological loss in weight $(5.41 \%)$ was recorded in fruits coated with 1.5 per cent guar gum and packed in perforated brown paper bags.

The mean values regarding storage period revealed that there was sharp increase in per cent physiological loss in weight from $6^{\text {th }}$ to $12^{\text {th }}$ day. The reduction in physiological loss in weight in coated fruits was probably due to the effect of these coating materials acting as a semi permeable barrier against oxygen, carbon dioxide, moisture and solute movement, thereby reducing respiration rate, water loss and oxidation reaction rates (Baldwin et al., 1999).

Table.1 Effect of different treatments and storage intervals on the physiological loss in weight $(\%)$ of litchi fruits

\begin{tabular}{|c|c|c|c|c|c|c|c|c|}
\hline Treatments & Day- & Day-2 & Day-4 & Day-6 & Day-8 & Day-10 & Day-12 & Mean A \\
\hline $\mathbf{T}_{\mathbf{0}}$ & 0.00 & 2.52 & 4.40 & 6.51 & 10.22 & 12.63 & 13.88 & 7.17 \\
\hline $\mathbf{T}_{\mathbf{1}}$ & 0.00 & 2.25 & 4.34 & 6.30 & 10.10 & 12.66 & 13.72 & 7.08 \\
\hline $\mathbf{T}_{\mathbf{2}}$ & 0.00 & 2.52 & 4.26 & 6.12 & 8.43 & 11.44 & 12.60 & 6.48 \\
\hline $\mathbf{T}_{\mathbf{3}}$ & 0.00 & 1.19 & 3.11 & 5.23 & 8.93 & 11.27 & 12.30 & 6.00 \\
\hline $\mathbf{T}_{\mathbf{4}}$ & 0.00 & 1.14 & 2.96 & 4.99 & 7.73 & 10.06 & 10.98 & 5.41 \\
\hline $\mathbf{T}_{\mathbf{5}}$ & 0.00 & 2.17 & 4.09 & 6.21 & 9.91 & 12.25 & 13.27 & 6.84 \\
\hline $\mathbf{T}_{\mathbf{6}}$ & 0.00 & 1.88 & 3.80 & 5.92 & 9.62 & 11.96 & 12.99 & 6.60 \\
\hline $\mathbf{T}_{\mathbf{7}}$ & 0.00 & 2.52 & 4.26 & 6.12 & 8.43 & 11.44 & 12.60 & 6.48 \\
\hline $\mathbf{T}_{\mathbf{8}}$ & 0.00 & 1.65 & 3.57 & 5.69 & 9.39 & 11.73 & 12.76 & 6.40 \\
\hline $\mathbf{T}_{\mathbf{9}}$ & 0.00 & 1.17 & 3.00 & 4.99 & 7.99 & 10.32 & 11.29 & 5.54 \\
\hline $\mathbf{T}_{\mathbf{1 0}}$ & 0.00 & 2.44 & 4.36 & 6.48 & 10.18 & 12.52 & 13.55 & 7.05 \\
\hline $\mathbf{T}_{\mathbf{1 1}}$ & 0.00 & 1.35 & 3.27 & 5.39 & 9.09 & 11.43 & 12.46 & 6.14 \\
\hline $\mathbf{T}_{\mathbf{1 2}}$ & 0.00 & 1.24 & 3.16 & 5.28 & 8.98 & 11.32 & 12.35 & 6.05 \\
\hline $\mathbf{T}_{\mathbf{1 3}}$ & 0.00 & 2.16 & 4.08 & 6.20 & 9.90 & 12.24 & 13.27 & 6.84 \\
\hline $\mathbf{T}_{\mathbf{1 4}}$ & 0.00 & 2.36 & 4.28 & 6.40 & 10.10 & 12.44 & 13.47 & 7.01 \\
\hline $\mathbf{T}_{\mathbf{1 5}}$ & 0.00 & 1.55 & 3.47 & 5.59 & 9.29 & 11.63 & 12.66 & 6.31 \\
\hline $\mathbf{T}_{\mathbf{1 6}}$ & 0.00 & 1.90 & 3.82 & 5.94 & 9.64 & 11.98 & 12.88 & 6.60 \\
\hline $\mathbf{T}_{\mathbf{1 7}}$ & 0.00 & 2.12 & 4.04 & 6.16 & 9.86 & 12.20 & 13.23 & 6.80 \\
\hline $\mathbf{M e a n}_{\mathbf{m}} \mathbf{F a c t o r s}$ & 0.00 & 1.90 & 3.79 & 5.86 & 9.32 & 11.75 & 12.79 & \\
\hline Factor(A) & & & $\mathbf{C . D .}$ & & & $\mathbf{S E}(\mathbf{m})$ \\
\hline Factor(B) & & & 0.14 & & & 0.05 & \\
\hline Factor (A X B) & & & 0.09 & & & 0.03 \\
\hline
\end{tabular}


Table.2 Effect of different treatments and storage intervals on fruit length (mm) of litchi

\begin{tabular}{|c|c|c|c|c|c|c|c|c|c|}
\hline Treatments & Day-0 & Day-2 & Day-4 & Day-6 & Day-8 & Day-10 & Day-12 & Mean A & Shrinkage \% \\
\hline $\mathbf{T}_{0}$ & 34.64 & 34.61 & 34.54 & 34.46 & 34.41 & 34.39 & 33.24 & 34.33 & 4.04 \\
\hline$T_{1}$ & 34.14 & 33.94 & 33.75 & 33.44 & 33.29 & 33.09 & 32.89 & 33.51 & 3.66 \\
\hline $\mathbf{T}_{2}$ & 34.41 & 34.24 & 34.03 & 33.73 & 33.57 & 33.40 & 33.19 & 33.80 & 3.55 \\
\hline $\mathbf{T}_{3}$ & 34.64 & 34.47 & 34.26 & 33.96 & 33.79 & 33.63 & 33.42 & 34.02 & 3.52 \\
\hline $\mathbf{T}_{4}$ & 34.71 & 34.66 & 34.50 & 34.36 & 34.23 & 34.05 & 33.86 & 34.34 & 2.45 \\
\hline $\mathbf{T}_{5}$ & 34.75 & 34.58 & 34.37 & 34.07 & 33.91 & 33.74 & 33.53 & 34.14 & 3.51 \\
\hline$T_{6}$ & 35.14 & 34.97 & 34.76 & 34.46 & 34.30 & 34.13 & 33.92 & 34.53 & 3.47 \\
\hline $\mathbf{T}_{7}$ & 34.27 & 34.10 & 33.90 & 33.60 & 33.43 & 33.26 & 33.05 & 33.66 & 3.56 \\
\hline$T_{8}$ & 34.31 & 34.14 & 33.93 & 33.63 & 33.47 & 33.30 & 33.10 & 33.70 & 3.53 \\
\hline$T_{9}$ & 35.10 & 34.93 & 34.72 & 34.42 & 34.26 & 34.09 & 33.88 & 34.49 & 3.48 \\
\hline$T_{10}$ & 34.71 & 34.54 & 34.33 & 34.03 & 33.87 & 33.70 & 33.49 & 34.10 & 3.51 \\
\hline$T_{11}$ & 34.67 & 34.50 & 34.30 & 34.00 & 33.83 & 33.66 & 33.48 & 34.06 & 3.43 \\
\hline$T_{12}$ & 35.32 & 35.15 & 34.95 & 34.65 & 34.48 & 34.31 & 34.10 & 34.71 & 3.45 \\
\hline$T_{13}$ & 34.51 & 34.34 & 34.14 & 33.84 & 33.67 & 33.51 & 33.32 & 33.91 & 3.45 \\
\hline$T_{14}$ & 34.21 & 34.04 & 33.84 & 33.54 & 33.37 & 33.20 & 32.99 & 33.60 & 3.57 \\
\hline$T_{15}$ & 35.10 & 34.93 & 34.73 & 34.43 & 34.26 & 34.09 & 33.88 & 34.49 & 3.48 \\
\hline$T_{16}$ & 34.73 & 34.56 & 34.36 & 34.06 & 33.89 & 33.72 & 33.51 & 34.12 & 3.51 \\
\hline$T_{17}$ & 35.40 & 35.23 & 35.03 & 34.73 & 34.56 & 34.39 & 34.18 & 34.79 & 3.45 \\
\hline Mean B & 34.71 & 34.55 & 34.36 & 34.08 & 33.92 & 33.76 & 33.50 & & \\
\hline \multicolumn{3}{|c|}{ Factors } & \multicolumn{4}{|c|}{ C.D. } & \multicolumn{3}{|c|}{ SE(m) } \\
\hline \multicolumn{3}{|c|}{ Factor(A) } & \multicolumn{4}{|c|}{0.54} & \multicolumn{3}{|c|}{0.19} \\
\hline \multicolumn{3}{|c|}{ Factor(B) } & \multirow{2}{*}{\multicolumn{4}{|c|}{0.33}} & \multicolumn{3}{|c|}{0.12} \\
\hline \multicolumn{3}{|c|}{ Factor (A X B) } & & & & & \multicolumn{3}{|c|}{0.51} \\
\hline
\end{tabular}

Table.3 Effect of different treatments and storage intervals on the fruit breadth $(\mathrm{mm})$ of litchi

\begin{tabular}{|c|c|c|c|c|c|c|c|c|c|}
\hline Treatments & Day-0 & Day-2 & Day-4 & Day-6 & Day-8 & Day-10 & Day-12 & Mean A & Shrinkage \% \\
\hline $\mathbf{T}_{0}$ & 32.09 & 31.98 & 31.78 & 31.48 & 31.28 & 31.11 & 30.00 & 31.39 & 6.51 \\
\hline$T_{1}$ & 32.08 & 31.97 & 31.77 & 31.47 & 31.27 & 31.11 & 30.02 & 31.39 & 6.42 \\
\hline $\mathbf{T}_{2}$ & 32.05 & 31.94 & 31.74 & 31.44 & 31.24 & 31.07 & 30.95 & 31.49 & 3.43 \\
\hline $\mathbf{T}_{3}$ & 32.07 & 31.96 & 31.76 & 31.46 & 31.26 & 31.09 & 30.97 & 31.51 & 3.43 \\
\hline $\mathbf{T}_{4}$ & 31.90 & 31.81 & 31.72 & 31.63 & 31.54 & 31.45 & 31.34 & 31.63 & 1.76 \\
\hline$T_{5}$ & 31.56 & 31.45 & 31.25 & 30.95 & 30.75 & 30.58 & 30.46 & 31.00 & 3.49 \\
\hline$T_{6}$ & 31.93 & 31.82 & 31.62 & 31.32 & 31.12 & 30.95 & 30.83 & 31.37 & 3.45 \\
\hline $\mathbf{T}_{7}$ & 32.14 & 32.03 & 31.83 & 31.53 & 31.33 & 31.16 & 31.04 & 31.58 & 3.42 \\
\hline$T_{8}$ & 32.33 & 32.22 & 32.02 & 31.72 & 31.52 & 31.35 & 31.23 & 31.77 & 3.40 \\
\hline$T_{9}$ & 32.08 & 31.97 & 31.77 & 31.47 & 31.27 & 31.11 & 30.99 & 31.52 & 3.40 \\
\hline$T_{10}$ & 32.07 & 31.91 & 31.75 & 31.59 & 31.43 & 31.27 & 31.12 & 31.59 & 2.96 \\
\hline$T_{11}$ & 31.55 & 31.44 & 31.24 & 30.94 & 30.74 & 30.58 & 30.45 & 30.99 & 3.49 \\
\hline$T_{12}$ & 31.90 & 31.79 & 31.59 & 31.29 & 31.09 & 30.92 & 30.80 & 31.34 & 3.45 \\
\hline$T_{13}$ & 32.42 & 32.31 & 32.11 & 31.81 & 31.61 & 31.44 & 31.34 & 31.86 & 3.33 \\
\hline$T_{14}$ & 32.06 & 31.95 & 31.75 & 31.45 & 31.25 & 31.09 & 30.97 & 31.50 & 3.40 \\
\hline$T_{15}$ & 31.88 & 31.77 & 31.57 & 31.27 & 31.07 & 30.90 & 30.78 & 31.32 & 3.45 \\
\hline$T_{16}$ & 32.09 & 31.98 & 31.78 & 31.48 & 31.28 & 31.11 & 30.99 & 31.53 & 3.43 \\
\hline$T_{17}$ & 31.85 & 31.74 & 31.54 & 31.24 & 31.04 & 30.88 & 30.76 & 31.29 & 3.42 \\
\hline Mean B & 32.00 & 31.89 & 31.70 & 31.42 & 31.23 & 31.07 & 30.84 & & \\
\hline \multicolumn{2}{|c|}{ Factors } & & \multicolumn{4}{|c|}{ C.D. } & \multicolumn{3}{|c|}{ SE(m) } \\
\hline \multicolumn{2}{|c|}{ Factor (A) } & & \multicolumn{4}{|c|}{0.46} & \multicolumn{3}{|c|}{0.17} \\
\hline \multirow{2}{*}{\multicolumn{2}{|c|}{$\begin{array}{c}\text { Factor (B) } \\
\text { Factor (A X B) }\end{array}$}} & & \multirow{2}{*}{\multicolumn{4}{|c|}{$\begin{array}{l}0.29 \\
\text { N/A }\end{array}$}} & \multicolumn{3}{|c|}{0.10} \\
\hline & & & & & & & \multicolumn{3}{|c|}{0.44} \\
\hline
\end{tabular}


Table.4 Effect of different treatments and storage intervals on the volume (ml) of litchi fruits

\begin{tabular}{|c|c|c|c|c|c|c|c|c|c|}
\hline Treatments & Day-0 & Day-2 & Day-4 & Day-6 & Day-8 & Day-10 & Day-12 & Mean A & \% decrease \\
\hline $\mathbf{T}_{\mathbf{0}}$ & 21.25 & 20.90 & 20.35 & 20.05 & 19.78 & 19.36 & 18.98 & 20.10 & $\mathbf{1 0 . 6 8}$ \\
\hline $\mathbf{T}_{\mathbf{1}}$ & 21.23 & 20.95 & 20.48 & 20.13 & 19.85 & 19.44 & 19.14 & 20.17 & $\mathbf{9 . 8 4}$ \\
\hline $\mathbf{T}_{\mathbf{2}}$ & 21.12 & 20.84 & 20.39 & 20.07 & 19.79 & 19.38 & 19.17 & 20.11 & 9.23 \\
\hline $\mathbf{T}_{\mathbf{3}}$ & 21.19 & 21.02 & 20.82 & 20.65 & 20.39 & 20.15 & 19.89 & 20.59 & 6.13 \\
\hline $\mathbf{T}_{\mathbf{4}}$ & 21.27 & 21.07 & 20.86 & 20.64 & 20.48 & 20.24 & 20.11 & 20.67 & $\mathbf{5 . 4 5}$ \\
\hline $\mathbf{T}_{\mathbf{5}}$ & 21.21 & 20.94 & 20.49 & 20.17 & 19.89 & 19.48 & 19.27 & 20.21 & 9.15 \\
\hline $\mathbf{T}_{\mathbf{6}}$ & 21.12 & 20.84 & 20.39 & 20.08 & 19.79 & 19.38 & 19.18 & 20.11 & 9.19 \\
\hline $\mathbf{T}_{\mathbf{7}}$ & 21.24 & 20.96 & 20.51 & 20.19 & 19.91 & 19.50 & 19.29 & 20.23 & 9.18 \\
\hline $\mathbf{T}_{\mathbf{8}}$ & 21.13 & 20.85 & 20.41 & 20.09 & 19.81 & 19.40 & 19.19 & 20.13 & 9.18 \\
\hline $\mathbf{T}_{\mathbf{9}}$ & 21.29 & 21.12 & 20.92 & 20.75 & 20.49 & 20.25 & 20.13 & 20.71 & $\mathbf{5 . 4 5}$ \\
\hline $\mathbf{T}_{\mathbf{1 0}}$ & 21.34 & 21.06 & 20.61 & 20.29 & 20.01 & 19.60 & 19.39 & 20.33 & 9.14 \\
\hline $\mathbf{T}_{\mathbf{1 1}}$ & 21.02 & 20.74 & 20.29 & 19.97 & 19.69 & 19.28 & 19.07 & 20.01 & 9.28 \\
\hline $\mathbf{T}_{\mathbf{1 2}}$ & 21.15 & 21.02 & 20.88 & 20.67 & 20.45 & 20.18 & 19.96 & 20.62 & 5.63 \\
\hline $\mathbf{T}_{\mathbf{1 3}}$ & 21.28 & 21.00 & 20.55 & 20.23 & 19.95 & 19.54 & 19.33 & 20.27 & 9.16 \\
\hline $\mathbf{T}_{\mathbf{1 4}}$ & 21.32 & 21.04 & 20.59 & 20.27 & 19.99 & 19.58 & 19.37 & 20.31 & 9.15 \\
\hline $\mathbf{T}_{\mathbf{1 5}}$ & 20.91 & 20.65 & 20.20 & 19.88 & 19.60 & 19.19 & 18.98 & 19.92 & 9.23 \\
\hline $\mathbf{T}_{\mathbf{1 6}}$ & 21.15 & 20.87 & 20.42 & 20.10 & 19.82 & 19.41 & 19.20 & 20.14 & 9.22 \\
\hline $\mathbf{T}_{\mathbf{1 7}}$ & 21.22 & 20.94 & 20.49 & 20.18 & 19.90 & 19.49 & 19.28 & 20.21 & 9.14 \\
\hline $\mathbf{M e a n}_{\mathbf{F}} \mathbf{2 1 . 1 9}$ & 20.93 & 20.54 & 20.25 & 19.98 & 19.60 & 19.39 & & \\
\hline Factors & & & $\mathbf{C . D .}$ & & & $\mathbf{S E}(\mathbf{m})$ & \\
\hline Factor(A) & & & 0.33 & & & 0.12 & \\
\hline Factor(B) & & & 0.21 & & & 0.07 & \\
\hline Factor (A X B) & & & N/A & & & 0.31 & \\
\hline
\end{tabular}

Table.5 Effect of different treatments and storage intervals on appearance of litchi fruits

\begin{tabular}{|c|c|c|c|c|c|c|c|c|}
\hline Treatments & Day-0 & Day-2 & Day-4 & Day-6 & Day-8 & Day-10 & Day-12 & Mean A \\
\hline $\mathbf{T}_{\mathbf{0}}$ & 9.00 & 6.42 & 6.14 & 5.92 & 5.80 & 5.69 & 4.45 & 6.20 \\
\hline$T_{1}$ & 9.00 & 7.02 & 6.68 & 6.34 & 5.78 & 5.34 & 4.20 & 6.34 \\
\hline $\mathbf{T}_{2}$ & 9.00 & 7.75 & 7.48 & 7.25 & 7.08 & 6.93 & 5.89 & 7.34 \\
\hline $\mathbf{T}_{3}$ & 9.00 & 7.54 & 7.28 & 7.05 & 6.88 & 6.73 & 5.69 & 7.17 \\
\hline $\mathbf{T}_{4}$ & 9.00 & 8.21 & 7.94 & 7.71 & 7.54 & 7.39 & 6.40 & 7.74 \\
\hline$T_{5}$ & 9.00 & 7.23 & 6.97 & 6.65 & 6.48 & 6.33 & 5.25 & 6.85 \\
\hline$T_{6}$ & 9.00 & 7.12 & 6.86 & 6.63 & 6.46 & 6.31 & 5.27 & 6.81 \\
\hline $\mathbf{T}_{7}$ & 9.00 & 7.52 & 7.26 & 7.03 & 6.86 & 6.71 & 5.67 & 7.15 \\
\hline $\mathbf{T}_{8}$ & 9.00 & 7.44 & 7.18 & 6.95 & 6.78 & 6.63 & 5.59 & 7.08 \\
\hline $\mathbf{T}_{9}$ & 9.00 & 8.05 & 7.79 & 7.56 & 7.39 & 7.24 & 6.25 & 7.61 \\
\hline$T_{10}$ & 9.00 & 7.82 & 7.56 & 7.33 & 7.16 & 7.01 & 5.97 & 7.41 \\
\hline$T_{11}$ & 9.00 & 7.74 & 7.48 & 7.25 & 7.08 & 6.93 & 5.89 & 7.34 \\
\hline$T_{12}$ & 9.00 & 7.98 & 7.72 & 7.49 & 7.32 & 7.17 & 6.20 & 7.56 \\
\hline$T_{13}$ & 9.00 & 6.98 & 6.72 & 6.49 & 6.32 & 6.17 & 5.13 & 6.69 \\
\hline$T_{14}$ & 9.00 & 7.47 & 7.21 & 6.98 & 6.81 & 6.66 & 5.62 & 7.11 \\
\hline$T_{15}$ & 9.00 & 7.23 & 6.97 & 6.76 & 6.59 & 6.44 & 5.40 & 6.91 \\
\hline$T_{16}$ & 9.00 & 7.04 & 6.78 & 6.55 & 6.38 & 6.23 & 5.19 & 6.74 \\
\hline$T_{17}$ & 9.00 & 7.13 & 6.87 & 6.64 & 6.47 & 6.32 & 5.28 & 6.82 \\
\hline Mean B & 9.00 & 7.43 & 7.16 & 6.92 & 6.73 & 6.57 & 5.52 & \\
\hline \multicolumn{2}{|c|}{ Factors } & & \multicolumn{3}{|c|}{ C.D. } & & \multicolumn{2}{|c|}{$\operatorname{SE}(\mathbf{m})$} \\
\hline \multirow{2}{*}{\multicolumn{2}{|c|}{$\begin{array}{l}\text { Factor(A) } \\
\text { Factor(B) }\end{array}$}} & & \multicolumn{3}{|c|}{0.10} & \multicolumn{3}{|c|}{0.03} \\
\hline & & & \multirow{2}{*}{\multicolumn{3}{|c|}{$\begin{array}{l}0.06 \\
0.25\end{array}$}} & \multirow{2}{*}{\multicolumn{3}{|c|}{0.09}} \\
\hline \multicolumn{2}{|c|}{ Factor (A X B) } & & & & & & & \\
\hline
\end{tabular}


Table.6 Effect of different treatments and storage intervals on the taste of litchi fruits

\begin{tabular}{|c|c|c|c|c|c|c|c|c|}
\hline Treatments & Day-0 & Day-2 & Day-4 & Day-6 & Day-8 & Day-10 & Day-12 & Mean A \\
\hline $\mathbf{T}_{\mathbf{0}}$ & 9.00 & 6.42 & 6.14 & 5.92 & 5.80 & 5.69 & 4.45 & $\mathbf{6 . 2 0}$ \\
\hline $\mathbf{T}_{\mathbf{1}}$ & 9.00 & 6.89 & 6.71 & 6.52 & 6.33 & 6.12 & 4.92 & $\mathbf{6 . 6 4}$ \\
\hline $\mathbf{T}_{\mathbf{2}}$ & 9.00 & 7.24 & 7.10 & 6.93 & 6.77 & 6.56 & 5.52 & 7.02 \\
\hline $\mathbf{T}_{\mathbf{3}}$ & 9.00 & 7.44 & 7.29 & 7.13 & 6.97 & 6.76 & 5.72 & 7.19 \\
\hline $\mathbf{T}_{\mathbf{4}}$ & 9.00 & 8.27 & 8.12 & 7.95 & 7.79 & 7.58 & 6.75 & $\mathbf{7 . 9 2}$ \\
\hline $\mathbf{T}_{\mathbf{5}}$ & 9.00 & 7.64 & 7.49 & 7.32 & 7.16 & 6.95 & 5.91 & 7.35 \\
\hline $\mathbf{T}_{\mathbf{6}}$ & 9.00 & 7.08 & 6.93 & 6.76 & 6.60 & 6.39 & 5.35 & 6.87 \\
\hline $\mathbf{T}_{\mathbf{7}}$ & 9.00 & 7.55 & 7.40 & 7.23 & 7.07 & 6.86 & 5.82 & 7.28 \\
\hline $\mathbf{T}_{\mathbf{8}}$ & 9.00 & 7.22 & 7.07 & 6.90 & 6.74 & 6.53 & 5.49 & 6.99 \\
\hline $\mathbf{T}_{\mathbf{9}}$ & 9.00 & 8.02 & 7.87 & 7.70 & 7.54 & 7.33 & 6.35 & $\mathbf{7 . 6 9}$ \\
\hline $\mathbf{T}_{\mathbf{1 0}}$ & 9.00 & 7.74 & 7.60 & 7.43 & 7.27 & 7.06 & 6.02 & 7.45 \\
\hline $\mathbf{T}_{\mathbf{1 1}}$ & 9.00 & 7.34 & 7.20 & 7.03 & 6.87 & 6.66 & 5.62 & 7.10 \\
\hline $\mathbf{T}_{\mathbf{1 2}}$ & 9.00 & 7.98 & 7.83 & 7.66 & 7.50 & 7.29 & 6.27 & 7.65 \\
\hline $\mathbf{T}_{\mathbf{1 3}}$ & 9.00 & 7.68 & 7.53 & 7.36 & 7.20 & 6.99 & 5.95 & 7.39 \\
\hline $\mathbf{T}_{\mathbf{1 4}}$ & 9.00 & 7.23 & 7.09 & 6.92 & 6.76 & 6.55 & 5.51 & 7.01 \\
\hline $\mathbf{T}_{\mathbf{1 5}}$ & 9.00 & 6.98 & 6.83 & 6.66 & 6.50 & 6.29 & 5.25 & 6.79 \\
\hline $\mathbf{T}_{\mathbf{1 6}}$ & 9.00 & 7.51 & 7.36 & 7.21 & 7.05 & 6.84 & 5.80 & 7.25 \\
\hline $\mathbf{T}_{\mathbf{1 7}}$ & 9.00 & 7.62 & 7.47 & 7.30 & 7.14 & 6.93 & 5.89 & 7.34 \\
\hline $\mathbf{M e a n}_{\mathbf{2}}$ & 9.00 & 7.44 & 7.28 & 7.11 & 6.95 & 6.74 & 5.70 & \\
\hline Factors & & & $\mathbf{C . D .}$ & & & $\mathbf{S E}(\mathbf{m})$ \\
\hline Factor (A) & & & 0.10 & & & 0.04 & \\
\hline Factor (B) & & & 0.06 & & & 0.02 & \\
\hline Factor (A X B) & & & 0.26 & & & 0.09 \\
\hline
\end{tabular}

Table.7 Effect of different treatments and storage intervals on the colour of litchi fruits

\begin{tabular}{|c|c|c|c|c|c|c|c|c|}
\hline Treatments & Day-0 & Day-2 & Day-4 & Day-6 & Day-8 & Day-10 & Day-12 & Mean A \\
\hline $\mathbf{T}_{\mathbf{0}}$ & 9.00 & 7.14 & 6.52 & 5.62 & 5.18 & 4.92 & 4.10 & 6.07 \\
\hline $\mathbf{T}_{\mathbf{1}}$ & 9.00 & 6.45 & 6.13 & 5.92 & 5.80 & 5.68 & 4.50 & 6.21 \\
\hline $\mathbf{T}_{\mathbf{2}}$ & 9.00 & 7.54 & 7.31 & 7.10 & 6.98 & 6.87 & 5.83 & 7.23 \\
\hline $\mathbf{T}_{\mathbf{3}}$ & 9.00 & 7.81 & 7.58 & 7.37 & 7.25 & 7.14 & 6.08 & 7.46 \\
\hline $\mathbf{T}_{\mathbf{4}}$ & 9.00 & 8.84 & 8.85 & 8.32 & 8.18 & 8.08 & 6.98 & $\mathbf{8 . 3 2}$ \\
\hline $\mathbf{T}_{\mathbf{5}}$ & 9.00 & 7.94 & 7.71 & 7.50 & 7.38 & 7.27 & 6.23 & 7.58 \\
\hline $\mathbf{T}_{\mathbf{6}}$ & 9.00 & 7.51 & 7.28 & 7.07 & 6.95 & 6.84 & 5.80 & 7.21 \\
\hline $\mathbf{T}_{\mathbf{7}}$ & 9.00 & 6.84 & 6.61 & 6.40 & 6.28 & 6.17 & 5.13 & 6.63 \\
\hline $\mathbf{T}_{\mathbf{8}}$ & 9.00 & 7.98 & 7.75 & 7.54 & 7.42 & 7.31 & 6.27 & 7.61 \\
\hline $\mathbf{T}_{\mathbf{9}}$ & 9.00 & 8.64 & 8.41 & 8.20 & 8.08 & 7.97 & 6.98 & $\mathbf{8 . 1 8}$ \\
\hline $\mathbf{T}_{\mathbf{1 0}}$ & 9.00 & 7.32 & 7.09 & 6.88 & 6.76 & 6.65 & 5.61 & 7.05 \\
\hline $\mathbf{T}_{\mathbf{1 1}}$ & 9.00 & 7.67 & 7.48 & 7.27 & 7.15 & 7.04 & 6.00 & 7.37 \\
\hline $\mathbf{T}_{\mathbf{1 2}}$ & 9.00 & 8.12 & 7.89 & 7.68 & 7.56 & 7.45 & 6.43 & 7.73 \\
\hline $\mathbf{T}_{\mathbf{1 3}}$ & 9.00 & 7.71 & 7.48 & 7.27 & 7.15 & 7.04 & 6.01 & 7.38 \\
\hline $\mathbf{T}_{\mathbf{1 4}}$ & 9.00 & 6.78 & 6.55 & 6.34 & 6.22 & 6.11 & 5.07 & 6.58 \\
\hline $\mathbf{T}_{\mathbf{1 5}}$ & 9.00 & 7.42 & 7.19 & 6.98 & 6.86 & 6.75 & 5.71 & 7.13 \\
\hline $\mathbf{T}_{\mathbf{1 6}}$ & 9.00 & 7.52 & 7.29 & 7.08 & 6.96 & 6.85 & 5.81 & 7.22 \\
\hline $\mathbf{T}_{\mathbf{1 7}}$ & 9.00 & 6.81 & 6.58 & 6.37 & 6.25 & 6.14 & 5.10 & 6.61 \\
\hline $\mathbf{M e a n}_{\mathbf{2}}$ & 9.00 & 7.56 & 7.32 & 7.05 & 6.91 & 6.79 & 5.76 & \\
\hline Factors & & & $\mathbf{C . D .}$ & & & $\mathbf{S E}(\mathbf{m})$ \\
\hline Factor (A) & & & 0.10 & & & & 0.04 & \\
\hline Factor (B) & & & 0.06 & & & & 0.02 & \\
\hline Factor (A X B) & & & 0.27 & & & \\
\hline
\end{tabular}


Table.8 Effect of different treatments and storage intervals on the Overall acceptability of litchi fruits

\begin{tabular}{|c|c|c|c|c|c|c|c|c|}
\hline Treatments & Day-0 & Day-2 & Day-4 & Day-6 & Day-8 & Day-10 & Day-12 & Mean A \\
\hline $\mathbf{T}_{\mathbf{0}}$ & 9.00 & 6.42 & 6.14 & 5.92 & 4.92 & 4.10 & 3.92 & 5.77 \\
\hline $\mathbf{T}_{\mathbf{1}}$ & 9.00 & 7.01 & 6.68 & 6.35 & 5.78 & 5.32 & 4.21 & 6.34 \\
\hline $\mathbf{T}_{\mathbf{2}}$ & 9.00 & 7.66 & 7.42 & 7.12 & 6.81 & 6.36 & 5.31 & 7.10 \\
\hline $\mathbf{T}_{\mathbf{3}}$ & 9.00 & 8.02 & 7.78 & 7.43 & 7.11 & 6.66 & 5.62 & 7.37 \\
\hline $\mathbf{T}_{\mathbf{4}}$ & 9.00 & 8.84 & 8.54 & 8.32 & 7.96 & 7.82 & 6.75 & 8.18 \\
\hline $\mathbf{T}_{\mathbf{5}}$ & 9.00 & 7.54 & 7.30 & 6.95 & 6.63 & 6.18 & 5.14 & 6.96 \\
\hline $\mathbf{T}_{\mathbf{6}}$ & 9.00 & 7.92 & 7.68 & 7.33 & 7.01 & 6.56 & 5.52 & 7.29 \\
\hline $\mathbf{T}_{\mathbf{7}}$ & 9.00 & 8.12 & 7.88 & 7.53 & 7.21 & 6.76 & 5.72 & 7.46 \\
\hline $\mathbf{T}_{\mathbf{8}}$ & 9.00 & 7.82 & 7.58 & 7.23 & 6.91 & 6.46 & 5.42 & 7.20 \\
\hline $\mathbf{T}_{\mathbf{9}}$ & 9.00 & 8.62 & 8.33 & 8.15 & 7.96 & 7.51 & 6.40 & 8.00 \\
\hline $\mathbf{T}_{\mathbf{1 0}}$ & 9.00 & 7.68 & 7.44 & 7.09 & 6.77 & 6.32 & 5.28 & 7.08 \\
\hline $\mathbf{T}_{\mathbf{1 1}}$ & 9.00 & 7.75 & 7.51 & 7.16 & 6.84 & 6.39 & 5.35 & 7.14 \\
\hline $\mathbf{T}_{\mathbf{1 2}}$ & 9.00 & 8.12 & 8.25 & 7.90 & 7.58 & 7.13 & 6.08 & 7.72 \\
\hline $\mathbf{T}_{\mathbf{1 3}}$ & 9.00 & 7.76 & 7.52 & 7.17 & 6.85 & 6.40 & 5.36 & 7.15 \\
\hline $\mathbf{T}_{\mathbf{1 4}}$ & 9.00 & 7.45 & 7.21 & 6.86 & 6.54 & 6.09 & 5.05 & 6.89 \\
\hline $\mathbf{T}_{\mathbf{1 5}}$ & 9.00 & 7.23 & 6.99 & 6.64 & 6.32 & 5.87 & 4.83 & 6.70 \\
\hline $\mathbf{T}_{\mathbf{1 6}}$ & 9.00 & 7.26 & 7.02 & 6.67 & 6.36 & 5.91 & 4.87 & 6.73 \\
\hline $\mathbf{T}_{\mathbf{1 7}}$ & 9.00 & 7.17 & 6.93 & 6.58 & 6.26 & 5.81 & 4.77 & 6.65 \\
\hline Mean B & 9.00 & 7.69 & 7.46 & 7.13 & 6.77 & 6.31 & 5.31 & \\
\hline Factors & & & $\mathbf{C . D}$ & & & SE(m) \\
\hline Factor (A) & & & 0.10 & & & 0.04 & 0.02 \\
\hline Factor (B) & & & 0.06 & & & & 0.10 \\
\hline
\end{tabular}

The above findings are in accordance with the findings of Bilawal et al., (2017), who reported a minimum decrease in physiological loss in weight in guava fruits coated with $2 \%$ calcium lactate, $2 \%$ xanthan gum and $4 \%$ glycerin during the 30 days of storage period.

\section{Fruit length}

Data depicted in table 2 indicates that packaging and edible coatings significantly affected the fruit length with the advancement of storage duration. The maximum shrinkage in length $(4.04 \%)$ was recorded in $\mathrm{T}_{0}$ i.e. fruits without coating and packaging, followed by $\mathrm{T}_{1}$ i.e.fruits without coating and packed in perforated brown paper bag (3.66\%), while the minimum shrinkage $(2.45 \%)$ was observed in treatment $\mathrm{T}_{4}$ i.e. fruits coated with guar gum $1.5 \%$ and packed in perforated brown paper bags. It might be due to the fact that coating the fruits with guar gum 1.5\% and packed in perforated brown paper bag effectively prevented the moisture loss and reduced respiration rate of the fruits. The above findings are in agreement with the findings of Dutta et al., (2016) and Tiwary (2011), who reported that coating of fruit significantly, prevented reduction in fruit length during the storage in ber and mango, respectively.

\section{Fruit breadth}

Fruit breadth gradually decreased in all the treatments with the advancement of the storage period (Table 3). The maximum shrinkage in breadth $(6.51 \%)$ was recorded in $\mathrm{T}_{0}$ i.e. fruits without coating and packaging, followed by $\mathrm{T}_{1}$ i.e. fruits without coating and packed in perforated brown paper bag $(6.42 \%)$, while the minimum shrinkage $(1.76 \%)$ was observed in treatment $\mathrm{T}_{4}$ i.e. fruits coated with guar gum $1.5 \%$ and packed 
in perforated brown paper bags, followed by $\mathrm{T}_{10}$ i.e. methyl cellulose (low viscosity) $0.5 \%$ and packed in perforated brown paper bag $(2.96 \%)$. The results showed that there was least fluctuation in fruit breadth in the treatment $\mathrm{T}_{4}$ indicating that coating the fruits with guar gum $1.5 \%$ along and packed in perforated brown paper bag effectively prevented the moisture loss and reduced respiration rate of the fruits. Cell degradation was also prevented which in turn facilitated reduced moisture loss and lesser respiratory gaseous exchange delaying senescence and decreasing the shrinkage percentage. The above findings are in agreement with the findings of Dutta et al., (2016), who reported that coating of fruit significantly prevented reduction in fruit breadth during the storage in ber.

\section{Fruit volume}

Packaging and edible coatings also affected the fruit volume with the advancement of storage duration (table 4). The maximum decrease in fruit volume percentage $(10.68 \%)$ was recorded in $\mathrm{T}_{0}$ i.e. fruits without coating and packaging, followed by $\mathrm{T}_{1}$ i.e. fruits without coating and packed in perforated brown paper bag $(9.48 \%)$, while there was the minimum change in fruit volume $(5.45 \%)$ in the treatment $\mathrm{T}_{4}$, followed by $\mathrm{T}_{9}$ i.e. $2 \%$ xanthum gum coated fruit packed in perforated brown paper bag $(5.45 \%)$. Coating and packaging help in reducing moisture loss from the fruits and thus prevents shrinkage and maintains better volume in the coated and packed fruits. Present study also revealed a gradual decrease in fruit volume with the increasing storage interval. As the storage interval increased, the reduction in per cent shrinkage also increased due to moisture loss. Decrease in fruit volume with the advancement of storage period has also been reported in mango (Tiwary, 2011).

\section{Appearance of litchi fruits}

Data in the table 5 revealed that the appearance of coated and uncoated litchi fruits packed in brown paper bag slowly decreased during storage period. The maximum appearance score (7.74) was obtained in the fruits coated with the guar gum $1.5 \%$ and packed in perforated brown paper bag $\left(\mathrm{T}_{4}\right)$, followed by $\mathrm{T}_{9}$ i.e. $2 \%$ xanthum gum coated fruit packed in perforated brown paper bag(7.61). The minimum score (6.20) was recorded in $\mathrm{T}_{0}$ i.e. fruits without coating and packaging, followed by $\mathrm{T}_{1}$ i.e.fruits without coating and packed in perforated brown paper bag (6.34). The results showed that there was minimum change in appearance of litchi fruits from day zero to day twelve of storage in the treatment $\mathrm{T}_{4}$ indicating that fruits coated with 1.5 per cent guar gum and packed in perforated brown paper bag effectively conserved the quality and appearance.

\section{Fruit taste}

Data presented in table 6 indicates that the coatings and packaging significantly affected the taste of fruits with the advancement of storage duration. Maximum score in fruit taste (7.92) was obtained in the fruits coated with 1.5 per cent guar gum and packed in perforated brown paper bag, followed by $\mathrm{T}_{9}$ i.e. $2 \%$ xanthum gum coated fruit packed in perforated brown paper bag (7.69).

The minimum score (6.20) was recorded in $\mathrm{T}_{0}$ (fruits without coating and packaging). The results showed that there was minimum change in taste of fruits from day Zero to day twelve of storage, in the treatment T4indicating that fruits coated with 1.5 per cent guar gum and packed in perforated brown paper bags effectively conserved the quality and taste of litchi fruits. 


\section{Fruit colour}

All the treatments exerted a significant influence on fruit colour (table 7). The highest colour score of litchi fruits (8.32) was recorded in fruits coated with 1.5 per cent guar gum and packed in perforated brown paper bag, followed by $\mathrm{T}_{9}$ i.e. $2 \%$ xanthum gum coated fruit packed in perforated brown paper bag (8.18), while the least score in colour (6.07) was recorded in $\mathrm{T}_{0}$ i.e. fruits without coating and packaging. Modified atmosphere created by the coating and packaging retarded ethylene production rate. Therefore, delayed ripening, chlorophyll degradation, anthocyanin accumulation and carotenoid synthesis, thus ultimately delayed colour change of fruits. The above results supported the findings of Brishti et al., (2013) and Tripathi and Dubey (2004), who found better retention in colour when papaya fruits were treated with Aloe vera base edible coatings.

\section{Overall Acceptability of Fruit}

In the present study, the overall acceptability of coated and uncoated litchi fruits packed in brown paper bag was slowly decreased during storage (table 8). Highest overall acceptability of fruits score (8.18) was recorded in $\mathrm{T}_{4}$ i.e.followed by $\mathrm{T}_{9}$ (8.00), while the minimum score (5.77) was recorded in $\mathrm{T}_{0}$ i.e. fruits without coating and packaging (control).These results supported the findings of Brishti et al., (2013)and Martinez et al., (2006) in papaya and cherry fruits, respectively.

In conclusion the pericarp browning is the major post-harvest problem of litchi fruits, which reduced commercial value of the fruit. Generally, visual quality was lost at ambient temperature when fruit were removed from storage as result of browning. We successfully optimized some physiological and sensory characteristics of litchi during storage by coating and packaging. By this study, there will be an alternative approach to prolong shelf-life of litchi fruits during postharvest.

\section{References}

Baldwin, E.A., Burns, J.K., Kazokas, W., Brecht, J.K., Hagenmaier, R.D., Bender, R.J. and Pesis, E. 1999. Effect of edible coatings with different permeability characteristics on mango (Mangifera indica $\mathrm{L}$.) ripening during storage. Postharvest Biology and Technology, 17(3): 215-226.

Baldwin, E.A., Nisperos-Carriedo, M.O., Baker, R.A., 1995. Use of edible coatings for lightly processed fruits and vegetables. Horticultural Science, 30: 35-38.

Bilawal, A., Hashmi, M.S., Zareen, S., Amir, M.N. and Khan, I. 2017. Effect of edible gum coating, glycerin and calcium lactate application on the postharvest quality of guava fruit. International Journal of Advanced Research and Publications, 1(5): 2327.

Brishti, F.H., Misir, J. and Sarker, A. 2013. Effect of bio preservatives on storage life of papaya fruit (Carica papaya L.). International Journal of Food Studies, 2: 126-136.

Dutta, P., Dey, K., Ghosh, A., Bhowmick, N. and Ghosh, A. 2016. Effect of edible coatings for enhancing shelf-life and quality in Ber (Zizyphus mauritiana Lamk.) fruits. Journal of Applied and Natural Science, 8(3): 1421-1426.

Huang, X.M., 2002. Lychee production in China. In: Papademetriou, M.K., Dent, F.J. (Eds.), Lychee Production in the Asia-Pacific Region. Food and Agricultural Organization of the United Nations, Bangkok, Thailand, 
pp. 41-54.

Jiang, Y.M. 2000. Role of anthocyanins, polyphenol oxidase and phenols in lychee pericarp browning. Journal of the Science of Food and Agriculture, 80: 305-310.

Jiang, Y.M., and Li, Y.B. 2003. Effects of low temperature acclimation on browning of litchi fruit in relation to shelf life. Journal of Horticultural Science and Biotechnology, 78: 437440.

Kumar, A., Singh, O. and Kohli, K. 2017. Post-harvest changes in functional and sensory properties of guava (Psidium guajava L. cv. Pant Prabhat) fruits as influenced by different edible coating treatments. Journal of Pharmacognosy and Phytochemistry, 6(6): 1109-1116.

Lin, B., Du, Y., Liang, X., Wangc, X., Wang, X. and Yang, J. 2011. Effect of chitosan coating on respiratory behavior and quality of stored litchi under ambient temperature. Journal of Food Engineering, 102: 94-99.

Mahajan, B.V.C., Dhatt, A.S., Dhatt, R.K. and Sharma, R.C. 2005. Effect of sulphur dioxide fumigation on the colour retention and quality of litchi cv. Calcuttia during cold storage. Haryana Journal of Horticultural Science, 32(1\&2): 47-49.

Martinez-Romero, D.F., Guillen, J.M., Valverde, M., Serrano, P., Zapata G., Castillo, and Valero, D. 2006. Horticoltura, -Revista-De-lndustria, -
Distribucion-y-socioecon $\sim$ mia-

Horticola. 195: 42-44.

Ruelas-Chacón, X., Contreras-Esquivel, J.C. and Montañez J.et al. 2017. "Water vapor permeabiliy, mecanical, optical, and sensorial properties of plasticized guar gum edible films," in Research Methodology in Food Science Integrated Theory and Practice, C. O. Mohan, E. Carvajal-Millan, and C. N. Ravishankar, (Eds.) Apple Academic Press, CRC Press, Taylor \& Francis Group, 2017.

Snedecor, G.W. and Cochran, W.G. 1987. Statistical Methods. Oxford and IBH Publishing Co. 66, Janpath, New Delhi-1

Tiwary, A.K. 2011. Effect of paraffin liquid and packaging materials on shelf life and quality of mango fruits cv. Dashehari. Thesis, Ph.D. Horticulture, G.B. Pant University of Agriculture and Technology, Pantnagar, $402 \mathrm{p}$.

Tripathi, P. and Dubey, N. 2004. Exploitation of natural products as an alternative strategy to control postharvest fungal rotting of fruit and vegetables. Postharvest Biology and Technology, 32: 235-245.

Zauberman, G., Ronen, R., Akerman, M., Weksler, A., Rot, I., and Fuchs, Y. 1991. Postharvest retention of the red colour of litchi fruit pericarp. Scientia Horticulture, 47: 89-97.

\section{How to cite this article:}

Shubham, N. K. Mishra, Ratna Rai, Ankit Dongariyal, Ravi Kumar and Tribhuwan Pratap. 2020. Effect of Edible Coating and Packaging on Physiological and Sensory Attributes of Litchi (Litchi chinensis Sonn.) Fruits. Int.J.Curr.Microbiol.App.Sci. 9(11): 517-526. doi: https://doi.org/10.20546/ijcmas.2020.911.063 